\title{
Effect of an education and mindfulness-based physical activity intervention for the promotion of positive body image in Lithuanian female students
}

\author{
Vaiva Balciuniene $^{1}$ (D) $\cdot$ Rasa Jankauskiene $^{2}$ (D) Migle Baceviciene $^{1}$ (D)
}

Received: 17 February 2021 / Accepted: 10 April 2021 / Published online: 20 April 2021

(c) The Author(s), under exclusive licence to Springer Nature Switzerland AG 2021

\begin{abstract}
Purpose The aim of this study was to test the effectiveness of an 8-week classroom education and mindfulness-based physical activity intervention for the promotion of positive body image in female students.

Methods A quasi-experimental study was carried out with 110 Lithuanian female students (mean age $21.5 \pm 3.5$, range 19-35 years). Thirty students voluntarily participated in the intervention programme and 80 students volunteered in the assessment-only control group. The intervention comprised five cognitive behavioural therapy classroom education sessions and mindfulness-based exercise (one exercise workout per week). The intervention group was evaluated with a pre-test and post-test and the control group completed measures at parallel times.

Results Compared with the control group participants, the intervention group participants reported greater improvements in positive body image and a reduction in body dissatisfaction and drive for thinness and internalisation of stereotyped beauty ideals, with medium to large effects. There were significant time $\times$ group interactions for pre-test to post-test changes in internalisation of beauty ideals, body area satisfaction, body dissatisfaction and body appreciation. In all cases, the interaction reflected greater pre-test to post-test changes in the intervention group compared with the control group, whose scores remained stable. A decrease in appearance orientation, overweight preoccupation, disordered eating and physical activity was observed in the control group, but the effect sizes were low.

Conclusion The preliminary findings of this study support the efficacy of cognitive behavioural therapy methods and mindfulness-based exercise intervention aimed to promote positive body image in student-aged women. Future studies should test the efficacy of the introduced programme in larger randomised samples of young women.
\end{abstract}

Level IV Evidence obtained from multiple time series with or without an intervention.

Keywords Positive body image $\cdot$ Mindful exercise $\cdot$ Intuitive eating $\cdot$ Positive embodiment $\cdot$ Body functionality $\cdot$ Intervention

Migle Baceviciene

migle75@gmail.com

Vaiva Balciuniene

balciuniene.vaiva@gmail.com

Rasa Jankauskiene

rasa.jankauskiene@1su.lt

1 Department of Physical and Social Education, Lithuanian Sports University, Sporto 6, 44221 Kaunas, Lithuania

2 Institute of Sport Science and Innovations, Lithuanian Sports University, Sporto 6, 44221 Kaunas, Lithuania

\section{Introduction}

Body dissatisfaction and disordered eating are prominent problems among higher education students worldwide [1]. The elevated prevalence of eating disorders in students compared with the general population has been observed in previous studies [1,2]. Recent studies have demonstrated that nearly $60 \%$ of students screened online were at risk for eating disorders or had an eating disorder [1,2]. The majority of students have not received treatment and one of the main reasons for that was a lack of a perceived need [2]. Therefore, timely prevention of body image concerns and disordered eating in the student population is a major public health priority [1-4]. 
Traditionally, universal intervention programmes have targeted negative body image and disordered eating and have promoted lifestyle-related behavioural changes in student-aged women. The findings from studies have revealed significant, small to moderate effects on eating disorder symptoms and risk factors [3]. Successful programmes are carried out in groups and involve dissonance-based intervention, cognitive behaviour therapy, media literacy education, self-esteem enhancement strategies and stress management skills [3, 5-10].

The development theory of embodiment (DTE) [11], which includes conceptualisation of positive body image and body functionality [12-14] has recently emerged. Researchers suggest that effective interventions should reduce negative outcomes of body image concerns and encourage positive and protective outcomes such as body appreciation, flexibility of body image, body functionality, positive attunement with exercise (AWE), intuitive eating and general well-being $[12,15,16]$. Positive body image is not simply the absence of body dissatisfaction or the end of the negative body image continuum [12]. It is an independent and multifaceted construct comprising three main components: appreciation of the body and its functionality, awareness and attentiveness to the body's needs and the ability to process body-related information in a self-protective way. It might also be described as the positive experience of an 'embodiment' [11,12].

An emphasis on building strengths and focusing on protective factors is important for successful interventions [17]. Universal programmes that aim to promote positive body image may be more attractive for the majority of students, especially those with low body image concerns and disordered eating, compared with the traditional emphasis on negative body image $[2,15]$. Studies suggest that not every student experiences body dissatisfaction or internalises the thin ideal, but all students can potentially benefit from healthier attitudes and practices in relation to their body and self $[2,17]$. Recently, the Norwegian healthy body image intervention programme for adolescents was implemented. The findings suggest that psychoeducation, media literacy and lifestyle changes [improving the experience of embodiment, the ability to reject exercise and nutritional myths and changing from an unhealthy to a healthy focus on the body] might foster positive body image $[18,19]$. Systematic reviews have shown that various interventions promoting positive body image in student-aged populations might also be effective [15-17]. However, only 13 interventions have aimed to foster positive body image in young women, and less than half of them have been implemented in Europe $[8,16]$. Testing interventions that promote positive body image in other than Western cultures is recommended [16]. Therefore, one of the aims of this study is to fill this gap.
Moreover, according to a systematic review [16], only two studies aiming to promote positive body image have included intervention-type exercise and tackled physical activity. Those studies reported significant improvements in weight concern in their target groups of women aged $41-52.5$ years $[20,21]$. However, there is a lack of universal intervention programmes that aim to promote positive body image and tackle physical activity in student-aged populations [8, 15-17]. A recent scoping review demonstrated that greater participation in sport is associated with more positive body image [22], but the direction of these associations is unclear. People with a more positive body image and greater body functionality might be more intensively engaged in physical activity and vice versa [14]. Thus, it is important to understand what impact physical activity has in positive body image $[12,22]$. There is lack of intervention studies to test this issue, and this study is aimed to add knowledge to this lacuna.

Evidence suggest that physical activity has a positive effect on body image only when it is focused on body functionality, but not when the goals are oriented towards body representation or appearance improvement [23-25]. Body functionality is a multifaceted construct that comprises everything that a body can do or is capable of doing [14]. People with high body functionality have internal body orientation focusing on what their body can do and how it feels rather than what body standards it meets and how it looks for themselves or others [26]. The newly developed conceptions of AWE [25] or intuitive exercise [27] states that individuals attuned to exercise have mindful attention, selfcompassion and self-acceptance; become more connected to the own body; and participate in safe physical activity in terms of quality and quantity. The exercise process is nonjudgmental, well balanced and joyful. It releases stress, gives pleasure, provides enjoyment and promotes positive body image [25, 27].

DTE $[11,28]$ suggests that in the physical freedom domain that centres on the physical care of the body, one of the protective factors that could be used in prevention programmes is pleasurable engagement in physical activities and the associated freedom from forced compliance with stereotyped appearance standards. These factors enhance the connection to one's body and participation in non-competitive sports, including those that support various body weights, comfortable clothing, hiking or wilderness and yoga. These activities contrast with joyless fitness activities aimed at body 'repair' and fitting harsh external standards $[11,25,29,30]$. These body functionality and AWE-oriented physical activity or fitness training interventions may promote positive body image by encouraging students to focus more on body functionality and less on their appearance $[14,28]$. In turn, these activities can increase internal motivation to exercise [31] and promote 
positive body image [25, 32]. Researchers have demonstrated that participation in body and mind integration that fosters physical activity, such as yoga, increases positive body image [33-35].

Based on the above-mentioned factors, the aim of this study was to test the effectiveness of an 8-week classroom education and mindfulness-based physical activity intervention for the promotion of the positive body image in Lithuanian female students. In this quasi-experimental pilot trial, we hypothesised that a programme encompassing education on media literacy, stress management, mindfulness, self-compassion, body functionality, healthy exercise, nutrition and mindfulness-based physical activity improves positive body image, decreases body dissatisfaction and disordered eating and has a positive impact on general physical activity in the intervention group compared with the assessment-only control group.

\section{Materials and methods}

\section{Participants}

We conducted a quasi-experimental trial with non- random allocation to the interventional and control groups with female students from various state and private universities and colleges located in Kaunas, the second largest city in Lithuania. In total, 288 students volunteered to participate in the pre-test, while drop-out led to a total of 110 (30 in the intervention and 80 in the control group) consenting students who participated in the post-intervention test (Fig. 1).

At baseline, the mean age was $21.5 \pm 3.5$ (range 19-35) years, while the mean body mass index (BMI) was $22.6 \pm 3.6$ (range 17.2-39.0) kg/m². After classifying participants into BMI categories, $6.4 \%$ of all study participants were underweight $\left(<18.5 \mathrm{~kg} / \mathrm{m}^{2}\right), 72.7 \%$ were normal weight $\left(18.5-24.9 \mathrm{~kg} / \mathrm{m}^{2}\right), 16.4 \%$ were overweight $(25.0-29.9 \mathrm{~kg} /$

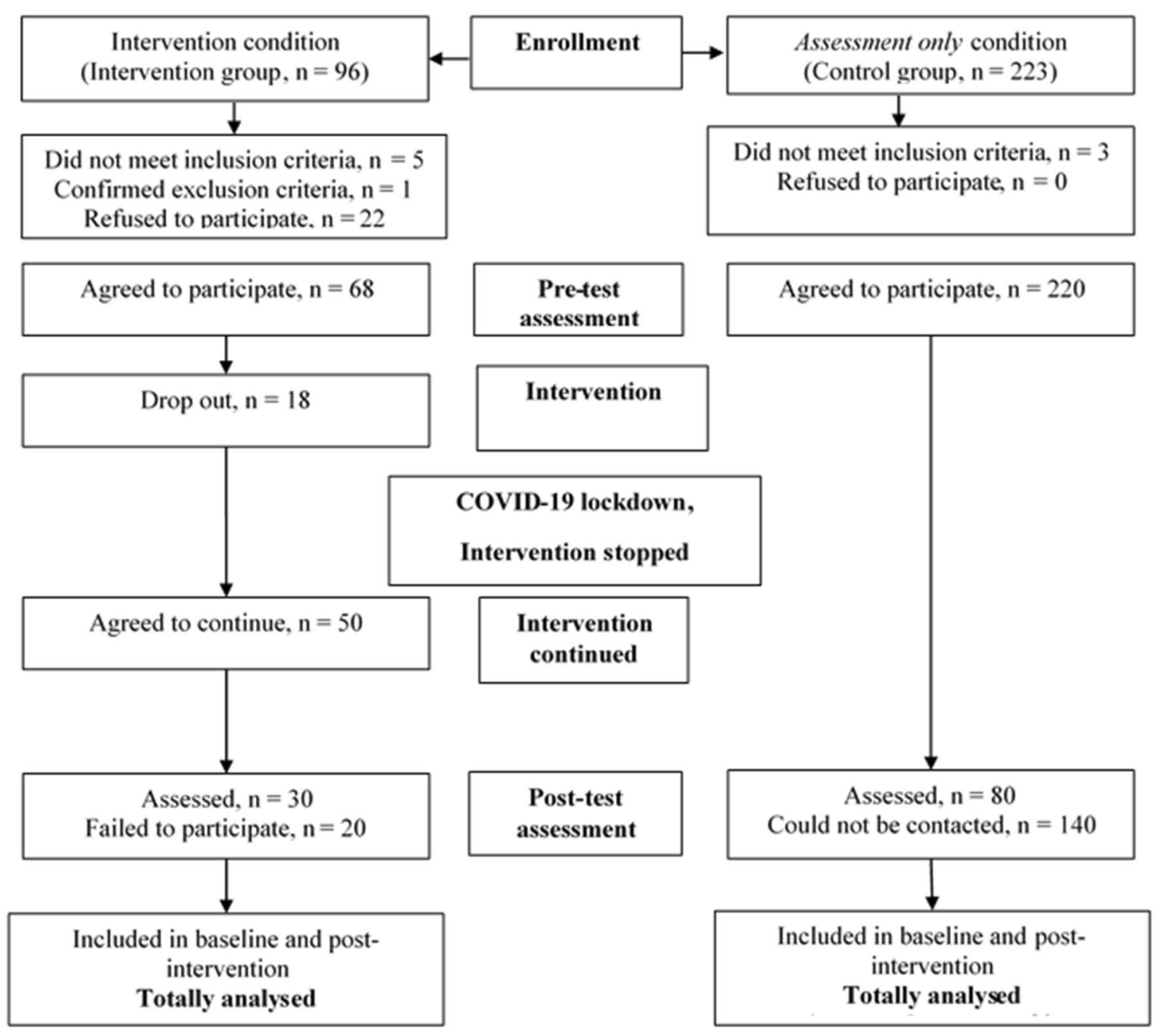

Fig. 1 Participant flow-chart 
$\left.\mathrm{m}^{2}\right)$ and $4.5 \%$ were obese $\left(\geq 30.0 \mathrm{~kg} / \mathrm{m}^{2}\right)$. There were no mean age and BMI differences between the intervention and control groups. The BMI, leisure-time exercise, body image concerns, disordered eating scores were very similar to the mean scores that were reported in previous research with a large Lithuanian student sample [36].

\section{Procedure}

Initially, we calculated that 25-34 participants should be included in the intervention and control groups when expecting a medium effect size (0.5-0.6), with $80 \%$ statistical power and at the $5 \%$ statistical significance level using two-sided statistical tests [37]. We planned to include 40 participants in both groups because of possible participant dropouts throughout the study. Due to time constraints and low student motivation to participate, as has been observed in previous studies [2,3], random allocation to intervention and control conditions was not possible.

Recruitment to the intervention group utilised multiple methods. First, we e-mailed study invitations to participants from our previous representative cross-sectional study on body image and health-related lifestyle. Then, we posted information through different social media accounts (universities, student representatives of universities and personal accounts of investigators). Finally, we invited female students to participate in a trial by leaving leaflets in university dormitories. The control group was selected using a nonprobability sampling method at the same universities and colleges in Kaunas. Participants were assigned to the control group, matching them to the intervention group's main baseline characteristics including age, study area and study cycle. The flow-chart of the study sample is presented in Fig. 1.

The newly developed 'Body and Mind' programme represents a universal intervention for student-aged females. Therefore, the study was presented for participants as an evaluation of the effects of physical activity on aspects of psychological well-being and a healthy lifestyle. Participants were not informed at the outset of the programme that the intervention targeted body image concerns. The inclusion criteria were (a) female gender, (b) studying at a university or college and (c) 19-35 years old. The exclusion criteria were existing musculoskeletal or other chronic conditions that would not enable participants to attend physical activity sessions.

The principal researcher (VB) conducted the study screening phone calls with interested participants to evaluate their eligibility. The most common reasons for dropping out of the study were conflicts with the academic schedule and COVID-19-associated constraints. As demonstrated in the participant flow-chart (Fig. 1), the final sample of student-aged females who voluntarily participated in the study was 30 participants in the intervention group and 80 in the control group.

The study procedures included a baseline survey and a post-intervention survey. The 8-week intervention programme was prolonged due to the COVID-19 lockdown and students' summer vacations. The first session of the intervention started on 7 March 2020. The COVID-19 lockdown lasted from 16 March to 11 May 2020. Thus, the intervention was stopped. We could not continue the intervention immediately after the lockdown (i.e. 12 May 2020) because of the enormous academic backlog caused by the lockdown and students' fears regarding their safety from COVID- 19 . Therefore, we decided to continue the intervention in the autumn semester, specifically from 3 October to 19 November 2020.

The four 4-h theoretical sessions with rest breaks took place in the university auditorium on 7 March and 3,10 and 24 October 2020. The fifth theoretical session was organised remotely on 7 November 2020 because of another COVID19 lockdown. Enrolled female students were divided into two groups of 15 students for participation in 1-h mindful exercise activities (a total of eight sessions per group; $1 \mathrm{~h}$ of exercise per week). The first group entered physical activity sessions every week on Tuesdays (10 March; 6, 13, 20 and 27 October; and 3, 10 and 17 November 2020), and the second group participated every week on Thursdays (12 March; 8, 15, 22 and 29 October; and 5, 12 and 19 November 2020). The six practical physical activity sessions took place in a health and fitness club, and the last two sessions were organised remotely because of the COVID-19 lockdown.

Enrolled participants completed the online baseline survey at the first session of the intervention and completed the post-intervention survey after the last session. All the measures included in the online survey were self-reported, and there was no time limit for completing the questionnaire.

\section{Ethical considerations and consent to participate}

We received ethical approval for the procedures in the quasiexperimental trial from the Committee for Social Sciences Research Ethics of the Lithuanian Sports University (protocol no. SMTEK-51, 09-12-2019). We also obtained written consent from female students to participate in the intervention programme.

\section{The 'Body and Mind' intervention programme}

A total of $24 \mathrm{~h}$ was allocated for the 8-week intervention programme. The curriculum consisted of 9 sessions: five 4-h interactive educational sessions and four 1-h exercise sessions (Table 1). Various previously recommended cognitive behavioural techniques adapted for the Lithuanian culture were used in the programme [6]. All sessions were 
Table 1 Description of the 'Body and Mind' intervention programme

\begin{tabular}{ll}
\hline Session & Description \\
\hline 1 & $\begin{array}{l}\text { The influence of the sociocultural environment that shapes our body's perception; appearance stereotypes and pressures to meet } \\
\text { appearance expectations; how to resist the pressure; the concept of body image and the importance of positive body image } \\
\text { Recognising emotions and stress management; practical stress management techniques }\end{array}$ \\
3 & $\begin{array}{l}\text { Strengthening of self-esteem, mindfulness, self-compassion and positive self-talk } \\
\text { Why does everyone need physical activity? What is exercise for the body and mind? }\end{array}$ \\
& $\begin{array}{l}\text { Physical activity and body functionality; mindful exercising; myths about physical activity and weight control } \\
\text { Recommendations for healthy eating; principles of intuitive eating }\end{array}$ \\
1 per week & $\begin{array}{l}\text { Slow \& Strong. A moderate-intensity strength and endurance workout, most of which consists of strength exercises for various } \\
\text { muscle groups. In this workout, the exercises are performed using only one's own body weight. Exercises performed during the } \\
\text { training strengthen the muscles of the whole body and the cardiovascular system }\end{array}$ \\
1 per week & $\begin{array}{l}\text { Nirvana Fitness. The Nirvana Body \& Mind system includes several efficient elements that provide a relaxing and mindful flow. } \\
\text { The programme includes uplifting music, simple to follow Pilates/yoga toning exercises and rhythmic breathing to detox the body } \\
\text { and experience the blissful state of Nirvana. The benefits of Nirvana are: enter the flow state, effective stress release, remove burn- } \\
\text { out symptoms, extra cell oxygenation and control muscle tone }\end{array}$ \\
$\begin{array}{c}\text { Total Body Workout. A full-body workout that ranges in intensity from low to high. During the training, strength, endurance, power, } \\
\text { coordination and balance are developed. Much attention is paid to the performance of functional movements and the improvement } \\
\text { of mobility. Various equipment is used, such as weights, rubber bands, balls, etc }\end{array}$ \\
$\begin{array}{c}\text { Functional Training. The type of session is designed to strengthen muscles and improve joint stability. During training, various } \\
\text { complex exercises are performed, and as many muscle groups as possible are involved in the work }\end{array}$
\end{tabular}

implemented by higher education lecturers and practitioners, specialists in their field; most of them were lecturers from Lithuanian Sports University. Interactive educational sessions were implemented on Saturdays in the auditorium of Lithuanian Sports University. Exercise workouts were implemented by highly qualified and experienced fitness instructors or coaches. Exercise workouts were implemented once per week on workdays in the 'Sporthouse' sport club located in Kaunas. This fitness club was chosen from various other health and fitness sports clubs because of the body-mindintegration-friendly sports infrastructure and policies (an all-body-friendly community, no 'fitspiration' pictures on the walls, fitness instructors did not wear tight apparel and there was relaxation and recovery after stress-based exercise activities). During the intervention, students in the control group followed their regular study curriculum.

\section{Measures}

Demographic data. In this study, female participants were asked to indicate their age and type of higher education institution (university or college).

Sociocultural Attitudes Towards Appearance Questionnaire-4 (SATAQ-4) [38] was used in this study to evaluate the role of appearance-related internalisation and sociocultural influence on body image. The 22-item SATAQ-4 contains five subscales where items are assessed on a 5-point Likert scale from 1 (definitely disagree) to 5 (definitely agree). A higher score indicates a greater level of appearance-related internalisation and a more significant sociocultural influence on body image. The five-item internalisation: thin/low body fat subscale describes an individual's preferences for a thin and low-fat body appearance. The fiveitem internalisation: athletic/muscular subscale evaluates an individual's beliefs about an athletic and muscular body appearance. The four-item pressure: family subscale indicates pressure from family members to adhere to social and cultural appearance expectations. The four-item pressure: peers subscale measures the extent of pressure from peers to match the dominant sociocultural standards of appearance. The four-item pressure: media subscale evaluates an individual's ability to resist media pressure of socially and culturally estimated appearance ideals. Good psychometric properties of the Lithuanian version of SATAQ-4 (LT-SATAQ-4) in a student population sample has been reported [39]. In the present sample at the baseline survey, the internal consistency (Cronbach's $\alpha$ ) for each subscale was as follows: total LT-SATAQ-4, 0.91; internalisation: thin/low body fat, 0.90; internalisation: athletic/muscular, 0.85 ; pressure: family, 0.90; pressure: peers, 0.93; and pressure: media, 0.98 .

The short version of the Multidimensional Body-Self Relations Questionnaire (MBSRQ-AS) [40] was used to evaluate the body image construct appearance-related components. The 34-item MBSRQ-AS consists of five subscales, where items are rated on a 5-point Likert scale from 1 (definitely disagree or very dissatisfied) to 5 (definitely agree or very satisfied). The seven-item appearance evaluation subscale describes an individual's perception of physical attractiveness or unattractiveness, where a higher score indicates general happiness about an individual's physical appearance. The 12-item appearance orientation subscale determines the degree of investment 
in an individual's appearance, where a lower score reflects general lower care about physical appearance. The nineitem body area satisfaction subscale reveals satisfaction or dissatisfaction with particular areas of the body, where a lower score defines general sadness with body size or the physical appearance of several body areas. The four-item overweight preoccupation subscale evaluates a construct reflecting dieting and eating restraint, weight vigilance and fat anxiety, where a higher score represents a greater preoccupation with being overweight. The two-item self-classified weight subscale explains an individual's weight perception and identification from underweight to overweight, where a higher score determines stronger beliefs that body weight is too high. A previous study demonstrated the reliability and validity of the Lithuanian version of the MBSRQ-AS (LT-MBSRQ-AS) in a student population sample [41]. In the present sample at the baseline survey, the internal consistency (Cronbach's $\alpha$ ) for each subscale was as follows: appearance evaluation, 0.89; appearance orientation, 0.75 ; body area satisfaction, 0.86 ; overweight preoccupation, 0.62 ; and self-classified weight, 0.84 .

The drive for thinness and body dissatisfaction were defined by the seven-item drive for thinness (DT) and 10-item body dissatisfaction (BD) subscales from the selfreport instrument Eating Disorder Inventory-3 (EDI-3) [42]. Answer choices for both subscales are provided on a 6-point Likert scale from 1 (never) to 6 (always), where a higher score indicates greater drive for thinness or higher body dissatisfaction. Research has supported good psychometric characteristics of DT and BD in Lithuanian nonclinical youth samples [43]. We purchased the required number of DT and BD copies from Psychological Assessment Resources, which holds the instrument's copyright. In the present sample at the baseline survey, the internal consistency (Cronbach's $\alpha$ ) for the subscales was as follows: LT-DT, 0.88; and LT-BD, 0.92.

Body appreciation concerning the body, body acceptance and resistance to media pressure about appearance ideals were evaluated by the 10-item self-report instrument Body Appreciation Scale-2 (BAS-2) [13]. The responses are based on a 5-point Likert scale from 1 (never) to 5 (always), where a higher score represents greater body appreciation. Research has confirmed the reliability and validity of the Lithuanian version of the BAS-2 (LT-BAS-2) in a sample of the youth population [43]. In the present sample at the baseline survey, the internal consistency (Cronbach's $\alpha$ ) for the LT-BAS-2 was 0.96 .

BMI was determined based on female students' selfreported data about their height and weight. Based on the BMI, participants were classified into four groups: underweight $\left(<18.5 \mathrm{~kg} / \mathrm{m}^{2}\right)$, normal weight $\left(18.5-24.9 \mathrm{~kg} / \mathrm{m}^{2}\right)$, overweight $\left(25.0-29.9 \mathrm{~kg} / \mathrm{m}^{2}\right)$ and obese $\left(\geq 30.0 \mathrm{~kg} / \mathrm{m}^{2}\right)$ [44].
Leisure-time physical activity in an average week was evaluated by the three-item self-report Godin-Shephard Leisure-Time Physical Activity Questionnaire (GSLTPAQ) [45]. The GSLTPAQ includes mild, moderate and strenuous physical activity during a week. The weekly global GSLTPAQ score provides a total metabolic equivalent for each intensity level. In the global score calculation formula, the number of bouts of mild exercise is multiplied by 3 , the number of bouts of moderate exercise is multiplied by 5 and the number of bouts of strenuous exercise is multiplied by 9. A higher score represents a higher level of leisure-time physical activity in an average week. In this study at the baseline survey, the global GSLTPAQ score was 47.8 (standard deviation $[\mathrm{SD}]=38.4$ ), and it ranged from 0 to 155 .

Disordered eating was defined by applying the 28-item self-report Eating Disorder Examination Questionnaire 6.0 (EDE-Q 6.0) [46]. Six open-ended questions provide the frequency data of the fundamental behavioural characteristics of eating disorders: objective binge eating, self-induced vomiting, laxative use and excessive exercise. The remaining 22 items comprise four subscales (restraint, eating concern, shape concern and weight concern) and indicate the severity of disordered eating. Answer options for the items in the subscales are given on a 7-point Likert scale from 0 (no days) to 6 (every day), where a higher score reflects greater disordered eating severity. The reliability and validity of the Lithuanian version of the EDE-Q 6.0 (LT-EDE-Q 6.0) have been confirmed in a student population [47]. In the present sample at the baseline survey, the internal consistency for the LT- EDE-Q 6.0 was rated as good $(\alpha=0.89)$.

Psychosocial stress was assessed by The Reeder Stress Inventory (RSI), measuring subjectively perceived stress, was used to determine psychosocial stress [48]. In the quasiexperimental trial, we used an adapted seven-item RSI (LTRSI) that was validated for use in the Lithuanian language [49]. The scale includes statements with responses based on a 4-point Likert scale from 1 (No, I disagree) to 4 (Yes, I agree). The trial participants had to indicate the answer for each of the statements that describe their feelings. The scores for all items are summed, and the general perceived stress score is obtained, which can range from 7 to 28 . A higher rating on the scale indicates greater perceived stress. In the present sample at the baseline survey, the internal reliability (Cronbach's $\alpha$ ) was 0.80 .

\section{Statistical analyses}

All data were first tested to determine whether they were normally distributed. To compare the baseline characteristics between the groups, normally distributed study variables were compared with independent Student's $t$-test and non-normally distributed study variables were compared with the Mann-Whitney $U$ test. To compare pre-test and 
post-test results, paired-sample statistics was used (normally distributed: paired-sample Student's $t$-test; non-normally distributed: Wilcoxon test). Data are presented as means and SDs alongside Cohen's $d$ effect sizes, which were classified as small (0.2-0.4), medium (0.5-0.6) or large $(\geq 0.7)$. Cohen's d effect size was calculated taking into account different sample sizes in the interventional and control groups. Cohen's d effect size was calculated taking into account different sample sizes in the interventional and control groups. Finally, two-way repeated measures analysis of variance (ANOVA) was conducted to test time and time $\times$ group interaction effects, with partial eta squared $\left(\eta^{2}\right)$ representing effect sizes. Internal consistency of study measures was tested by Cronbach's $\alpha$. The significance threshold was set at $p<0.05$. Statistical analyses were carried out using IBM SPSS Statistics 26 (IBM Corp., Armonk, NY, USA).

\section{Results}

Table 2 presents descriptive statistics of the study variables at baseline. There were only a few differences between the intervention and control groups. Specifically, the intervention group showed a higher level of perceived media pressures and a lower leisure-time exercise score compared with the control group.

Comparing the post-test with the pre-test, the body image concerns of the intervention group decreased: the SATAQ-4 total score and the thin/low body fat and muscular/athletic body internalisation subscale scores decreased with medium effect sizes (Table 3 ). There was no change in the perceived pressures subscales. Moreover, the MBSRQ-AS appearance evaluation and body area satisfaction subscales increased with a medium to large effect size, while the score in appearance orientation decreased (large effect size). The most important findings were observed in the body dissatisfaction and body appreciation scales, namely a decline in body dissatisfaction, drive for thinness and disordered eating behaviours and improvement in positive body image, all
Table 2 Comparison of the study variables at baseline between the intervention and control groups

\begin{tabular}{|c|c|c|c|c|}
\hline \multirow[t]{2}{*}{ Study variables } & \multirow{2}{*}{$\begin{array}{l}\text { Control group }(n=80) \\
\text { M (SD) }\end{array}$} & \multirow{2}{*}{$\begin{array}{l}\text { Intervention } \\
\text { group }(n=30) \\
\mathrm{M}(\mathrm{SD})\end{array}$} & \multirow[t]{2}{*}{$p$} & \multirow[t]{2}{*}{$d$} \\
\hline & & & & \\
\hline \multicolumn{5}{|l|}{$S A T A Q-4$} \\
\hline Total & $2.32(0.74)$ & $2.54(0.69)$ & 0.155 & - \\
\hline Thin/low body fat internalisation & $3.96(0.90)$ & $3.92(0.87)$ & 0.798 & - \\
\hline Muscular/athletic body internalisation & $2.55(0.88)$ & $2.86(0.99)$ & 0.139 & - \\
\hline Pressures: family & $1.81(1.12)$ & $1.82(1.00)$ & 0.645 & - \\
\hline Pressures: peers & $1.65(0.95)$ & $1.72(0.83)$ & 0.210 & - \\
\hline Pressures: media & $2.55(1.48)$ & $3.33(1.34)$ & 0.021 & -0.2 \\
\hline \multicolumn{5}{|l|}{$M B S R Q-A S$} \\
\hline Appearance evaluation & $3.18(0.92)$ & $3.30(0.85)$ & 0.533 & - \\
\hline Appearance orientation & $3.63(0.55)$ & $3.74(0.52)$ & 0.302 & - \\
\hline Body area satisfaction & $3.23(0.84)$ & $3.22(0.54)$ & 0.909 & - \\
\hline Overweight preoccupation & $2.29(0.82)$ & $2.08(0.88)$ & 0.225 & - \\
\hline Self-classified weight & $3.27(0.71)$ & $3.03(0.82)$ & 0.171 & - \\
\hline \multicolumn{5}{|l|}{ Body dissatisfaction and body appreciation } \\
\hline EDI: body dissatisfaction & $3.43(1.36)$ & $3.38(1.22)$ & 0.868 & - \\
\hline EDI: drive for thinness & $2.85(1.25)$ & $2.84(1.30)$ & 0.987 & - \\
\hline Body appreciation (BAS-2) & $3.36(1.06)$ & $3.48(0.78)$ & 0.521 & - \\
\hline \multicolumn{5}{|l|}{ Body mass index and lifestyle } \\
\hline Body mass index, $\mathrm{kg} / \mathrm{m}^{2}$ & 22.79 (3.68) & $21.93(3.23)$ & 0.248 & - \\
\hline Leisure-time exercise score & $53.85(41.42)$ & $31.63(21.99)$ & 0.022 & -0.2 \\
\hline Disordered eating (EDEQ-6) & $1.56(1.11)$ & $1.50(1.02)$ & 0.917 & - \\
\hline Stress score & $20.01(4.16)$ & $18.97(4.82)$ & 0.299 & - \\
\hline
\end{tabular}

$B A S-2$ body appreciation scale-2, $d$ Cohen's $d$ effect size, EDEQ-6 eating disorder examination questionnaire-6, $E D I$ eating disorder inventory, $M$ mean, $M B S R Q-A S$ multidimensional body-self relations questionnaire-appearance scales, $p$ statistical significance, $S A T A Q-4$ sociocultural attitudes towards appearance questionnaire- $4, S D$ standard deviation 
Table 3 Comparison of pre-test and post-test values for the intervention group $(n=30)$

\begin{tabular}{lllll}
\hline Study variables & Pre-test & Post-test & $p$ & $d$ \\
& M (SD) & M (SD) & & \\
\hline SATAQ-4 & & & & \\
Total & $2.54(0.69)$ & $2.26(0.79)$ & 0.004 & 0.6 \\
Thin/low body fat internalisation & $3.92(0.87)$ & $3.51(0.85)$ & 0.006 & -0.4 \\
Muscular/athletic body internalisation & $2.86(0.99)$ & $2.43(1.03)$ & 0.014 & 0.5 \\
Pressures: family & $1.82(1.00)$ & $1.72(0.85)$ & 0.266 & - \\
Pressures: peers & $1.72(0.83)$ & $1.69(1.01)$ & 0.574 & - \\
Pressures: media & $3.33(1.34)$ & $3.10(1.34)$ & 0.296 & - \\
MBSRQ-AS & & & & \\
Appearance evaluation & $3.30(0.85)$ & $3.55(1.01)$ & 0.007 & 0.5 \\
Appearance orientation & $3.74(0.52)$ & $3.44(0.53)$ & $<0.001$ & 0.9 \\
Body area satisfaction & $3.22(0.54)$ & $3.59(0.86)$ & 0.001 & 0.7 \\
Overweight preoccupation & $2.08(0.88)$ & $1.83(0.88)$ & 0.050 & -0.3 \\
Self-classified weight & $3.03(0.82)$ & $3.00(0.64)$ & 0.690 & - \\
Body dissatisfaction and body appreciation & & & & \\
EDI: body dissatisfaction & $2.84(1.30)$ & $2.33(1.20)$ & 0.003 & -0.4 \\
EDI: drive for thinness & $3.38(1.22)$ & $2.71(1.28)$ & $<0.001$ & 1.1 \\
Body appreciation (BAS-2) & $3.48(0.78)$ & $3.84(0.90)$ & 0.001 & 0.7 \\
Body mass index and lifestyle & & & & -0.3 \\
Body mass index, kg/m ${ }^{2}$ & $21.93(3.23)$ & $21.53(3.17)$ & 0.040 & -0.3 \\
Leisure-time exercise score & $31.63(21.99)$ & $37.67(31.59)$ & 0.447 & - \\
Disordered eating (EDEQ-6) & $1.50(1.02)$ & $1.16(1.20)$ & 0.034 & -0.3 \\
Stress score & $18.97(4.82)$ & $17.67(4.75)$ & 0.067 & - \\
\hline
\end{tabular}

$B A S-2$ body appreciation Scale-2, $d$ Cohen's d effect size, EDEQ-6 eating disorder examination questionnaire-6, $E D I$ eating disorder inventory, $M$ mean, $M B S R Q-A S$ multidimensional body-self relations questionnaire-appearance scales, $p$ statistical significance, SATAQ-4 sociocultural attitudes towards appearance questionnaire- $4, S D$ standard deviation with medium to high effect sizes. There was no significant change in the leisure-time exercise score.

In the control group, there was also some reduction in body image concerns, with small effect sizes, at the posttest. Specifically, the appearance orientation, overweight preoccupation and disordered eating behaviour scores were decreased (Table 4). In addition, the leisure-time exercise level significantly decreased.

Table 5 presents the combined time $\times$ group interaction effect on the study variables. We observed a decrease in body image concerns and disordered eating behaviours in intervention and control groups. It is important to notice that the significant time $\times$ group interaction effect for general internalisation of stereotyped body ideals, body area satisfaction, body appreciation and body dissatisfaction demonstrated a more marked change in the intervention group as a result of the educational programme. The interaction effect in the BMI and leisure-time exercise revealed a significant decline in BMI for the intervention group and a reduction in leisure-time exercise for the control group. The time $\times$ group interaction did not reach significance for thin and muscular ideal internalisation, appearance evaluation and orientation, overweight preoccupation and self-classified body weight, drive for thinness or disordered eating. Graphs illustrating the main interaction effects can be found in the supplementary files.

\section{Discussion}

In this study, we evaluated the efficacy of the newly developed universal intervention programme 'Body and Mind' among female university students in Lithuania. The intervention programme positively promoted positive body image, decreased internalisation of stereotyped sociocultural beauty ideals and body image concerns. This study makes a novel contribution since it is important to develop universal programmes that aim to promote positive body image and a healthy lifestyle in university-aged students [16]. Evidence shows that students who have moderate body image concerns usually do not engage in eating disorders intervention programmes since they do not perceive the need for it [2]. But all of them need protective education for the development of positive body image, useful knowledge, attitudes and skills of healthy nutrition and health-enhancing physical activity. Emphasis on building strengths rather than 
Table 4 Comparison of the pretest and post-test values for the control group $(n=80)$

\begin{tabular}{lllll}
\hline Study variables & Pre-test & Post-test & $p$ & $d$ \\
& M (SD) & M (SD) & & \\
\hline SATAQ-4 & & & & \\
Total & $2.32(0.74)$ & $2.30(0.78)$ & 0.752 & - \\
Thin/low body fat internalisation & $3.96(0.90)$ & $3.83(0.84)$ & 0.087 & - \\
Muscular/athletic body internalisation & $2.55(0.88)$ & $2.45(0.78)$ & 0.296 & - \\
Pressures: family & $1.81(1.12)$ & $1.95(1.08)$ & 0.117 & - \\
Pressures: peers & $1.65(0.95)$ & $1.67(1.02)$ & 0.555 & - \\
Pressures: media & $2.55(1.48)$ & $2.61(1.49)$ & 0.703 & - \\
MBSRQ-AS & & & & \\
Appearance evaluation & $3.18(0.92)$ & $3.30(0.95)$ & 0.078 & - \\
Appearance orientation & $3.63(0.55)$ & $3.48(0.59)$ & 0.002 & 0.4 \\
Body area satisfaction & $3.23(0.84)$ & $3.33(0.81)$ & 0.118 & - \\
Overweight preoccupation & $2.29(0.82)$ & $2.12(0.82)$ & 0.006 & -0.2 \\
Self-classified weight & $3.27(0.71)$ & $3.26(0.65)$ & 0.880 & - \\
Body dissatisfaction and body appreciation & & & & \\
EDI: body dissatisfaction & $2.85(1.25)$ & $2.67(1.17)$ & 0.060 & - \\
EDI: drive for thinness & $3.43(1.36)$ & $3.28(1.26)$ & 0.070 & - \\
Body appreciation (BAS-2) & $3.36(1.06)$ & $3.41(1.06)$ & 0.469 & - \\
Body mass index and lifestyle & & & & - \\
Body mass index, kg/m ${ }^{2}$ & $22.79(3.68)$ & $22.93(4.01)$ & 0.291 & - \\
Leisure-time exercise score & $53.85(41.42)$ & $43.94(33.62)$ & 0.016 & -0.2 \\
Disordered eating (EDEQ-6) & $1.56(1.11)$ & $1.32(1.09)$ & 0.002 & -0.2 \\
Stress score & $20.01(4.16)$ & $19.61(4.71)$ & 0.337 & - \\
\hline
\end{tabular}

$B A S-2$ body appreciation scale-2, $d$ Cohen's d effect size, EDEQ-6 eating disorder examination questionnaire-6, EDI eating disorder inventory, $M$ mean, $M B S R Q-A S$ multidimensional body-self relations questionnaire-appearance scales, $p$ statistical significance, $S A T A Q-4$ sociocultural attitudes towards appearance questionnaire-4, $S D$ standard deviation reduction of not yet fully developed risks is important in intervention programmes $[9,15]$.

Our sample represented healthy young women with low body image concerns that reflect the tendencies which were observed in other large samples of Lithuanian female students [36, 41]. At baseline, we found no significant differences between intervention and control groups except for a higher level of perceived media pressures and lower physical activity level in the intervention group. The present universal intervention promoted a post-intervention effect, namely increasing positive body image (body appreciation, appearance evaluation and body area satisfaction) and decreasing negative body image (body dissatisfaction and drive for thinness) and internalisation of sociocultural stereotyped beauty ideals, all with medium to large effect sizes. These variables did not significantly change in the control group when comparing the pre-test and post-test results. Following prior recommendations [50], we found time $\times$ group interactions in general internalisation of beauty ideals, body area satisfaction, body dissatisfaction, body appreciation and BMI. However, the changes were larger in the intervention compared with the control group, whose scores remained stable or changed insignificantly.
These results are in line with the previous studies reporting that education based on cognitive behavioural techniques using media literacy education, cognitive-dissonance-based strategies, psychoeducation, self-esteem enhancement strategies, improving stress management skills, discussing healthy exercise and eating might decrease negative body image $[3,5-8,10,17]$. These results are consistent with studies suggesting that increasing positive embodiment through body functionality and mindfulness might promote positive body image in adults and adolescents [15, 16, 18, 19]. A mindfulness-based exercise programme was one of the main components of the present intervention. The results of this study are in line with the concept of AWE [25] and the conception of intuitive exercise [27]. These theories indicate that mindful exercises can help to build or restore body and mind integrity and body functionality and provide positive embodiment through decreased appearance-oriented exercise motivation and body objectification. We asked our highly qualified fitness instructors to emphasise relaxation, body and mind integration and internal goals for exercise, and to avoid any comments on external outcomes of exercise such as appearance, fitness standards or body 


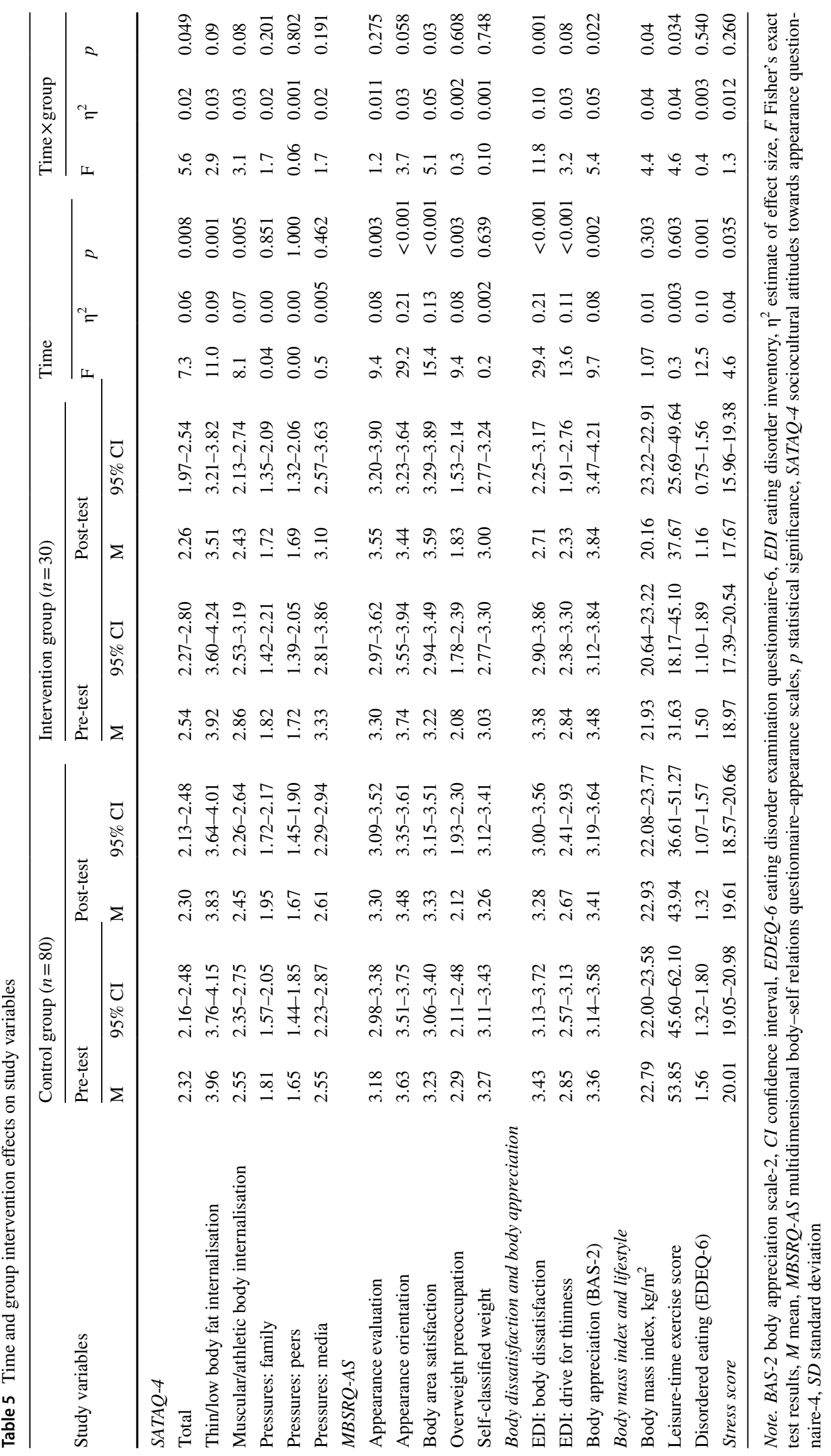


weight when implementing the workouts. Following prior recommendations [51], fitness coaches were also asked to focus on function, strength and personal improvement; facilitate participants' enjoyment and feelings of joy and competence; and teach participants to rely on their body feelings during exercise rather than external cues such as energy expenditure and exercise time [27]. Moreover, the exercise programme was implemented in a health and fitness club that follows body-and-mind-integration-based policies. Previous studies have demonstrated that those factors are important for body image in exercisers $[29,51]$.

Our study adds to the knowledge that attuned, intuitive or mindful exercise is an important variable that promotes positive body image in young women $[25,27,33,52,53]$. Some studies showed [35] that compared with controls participants, individuals performing yoga reported significant increases in body appreciation and body satisfaction at posttest and at 4-week follow-up. The novelty of this study is that the high variety of fitness activities [not only yoga], including strength and endurance workouts, Nirvana fitness, total body workouts and functional training, might help to promote positive body image in female students if exercise is mindful and oriented towards body functionality or attunement. This goes in line with the previous recommendations [51]. This pilot study adds to the knowledge that it is important to test various fitness activities and sports with specially trained fitness instructors or coaches to understand the impact of a wide variety of sports and physical activities on the development of positive body image in women. Studies in belly dancers, athletes and various sports involving adolescents showed the greater positive body image compared physical activity not involved students [54-56]. However, the present experimental study adds to the collected knowledge to understand the direction of these associations.

The 'Body and Mind' intervention programme is comprehensive, consisting of several sessions of class-based education (one month) and mindfulness exercises (one month with one exercise workout per week). Therefore, it was impossible to make any conclusions on the separate impact of education, exercise per se or various exercise types on positive body image. Because the number of intervention and control group participants was small, it was also impossible to understand the mechanisms underlying the changes in body image noted in the intervention group. This might be considered as a major limitation of this study. However, developing interventions that target multiple components of positive body image has been encouraged [16]. In this study, we aimed to test the impact of a universal educational programme on positive body image in female students and included exercise as the main part of the programme. In future studies aimed to understand fundamentally the influence of physical activity on positive body image, we recommend creating interventions that target physical activity alone without mixing it with the other interventions (e.g. education).

Of note, there was a decrease in negative body image in the control group between the pre-test and post-test. Specifically, appearance orientation, overweight preoccupation and disordered eating significantly decreased alongside physical activity, all with small effect sizes. These results might be explained by the COVID-19 lockdown that started immediately after the pre-test and lasted two months. Common concerns associated with the COVID-19 situation lasted all summer, and new cases of COVID-19 drastically started to spread in September 2020, a phenomenon that led to a second lockdown in Lithuania. Thus, we speculate that the body image changes in the control group might be related to the lockdown. These findings contradict the assumptions that the COVID-19 lockdown might negatively impact body image $[57,58]$. We observed that body image concerns and disordered eating also decreased in the intervention group [except overweight preoccupation and physical activity]. Therefore, because changes were seen in both groups, it is impossible to conclude whether the changes in intervention group were caused by the COVID-19 lockdown or the intervention.

In this study, we found that the BMI of the intervention group participants decreased from the pre-test to the posttest (low effect size) while the BMI of the control group was stable. Those results might be explained by increased energy expenditure in the intervention group. Moreover, the baseline leisure-time exercise score was significantly lower in the intervention group. The BMI decrease might be also related to possible changes in eating habits. Teaching on healthy eating and introducing intuitive eating principles were integrated into the educational program. Unfortunately, in this study, we did not assess changes in eating habits and intuitive eating, therefore, we can only speculate about the possible under-related mechanisms between the positive body image, healthy eating and BMI. Finally, based on the weight-inclusive approach to health, [59] reducing BMI was not the focus of the present intervention. Rather, it was one of the outcomes of the present intervention suggesting that the promotion of positive body image might be effective in overweight and obesity prevention.

Obesity and eating disorders share common risk factors such as body image concerns, disordered eating, depression, lack of physical activity and/or dysfunctional exercise. Therefore, the implementation of integrated programmes that tackle the prevention of obesity and eating disorders, is recommended $[15,60,61]$. The findings of this study have important implications for practice. Since the majority of universities students need education on positive body image, healthy eating, physical activity and sedentary lifestyle, developing and implementation of universal intervention programmes that focus on positive body image and integrate teaching about a healthy lifestyle is of great importance. 
Based on the findings of this study, we recommend implementing theoretical teaching about physical activity also including active students' participation in practical mindfulness-based physical activity workouts. This might help to foster positive body image, prevent disordered eating, to increase physical activity literacy and mindfulness-based exercising skills that might be helpful for lifelong physical activity and maintaining healthy body weight.

This study has important limitations that should be discussed. The main weakness is the lack of randomisation. As previously mentioned, randomisation to intervention or control groups was not possible due to time constraints, enormous academic loads following the first COVID-19 lockdown and lack of student motivation to participate in the study. A lack of student motivation has been reported in previous studies [2,3]. Another limitation is the small number of participants in the intervention and control groups. Hence, there was a lack of statistical power and, most importantly, the inability to understand the mechanisms underlying the changes in positive body image and other important variables of this study. Thus, future studies should test this programme with larger sample sizes. Another important limitation is implementation of the intervention during the COVID-19 pandemic, specifically the forced stop of the programme with a long period without intervention between the pre-test and post-test assessments. This phenomenon also led to significant drop-out of participants in both the intervention and control groups. We speculate that in this study we had two interventions: one for the intervention group (educational programme and COVID-19 lockdown) and one for the control group (COVID-19 lockdown). Therefore, the interpretation of the results is limited and the programme's efficacy should be re-tested in normal conditions. Finally, a follow-up assessment is necessary to understand the persistence of the changes in positive body image and related variables in young women. However, because the positive body image topic is relatively new, some of the intervention studies tackling positive body image lack follow-up data [16]. In sum, the results of this study should be interpreted with caution because of its limitations and because it was implemented in force majeure conditions.

This study has important strengths. The first strength is the use of a sound theoretical background. The educational programme was developed in accordance with recommended cognitive behavioural techniques for improving body image [6]. Furthermore, the exercise-related part of the programme was based on the concepts of AWE and intuitive exercise. To the best our knowledge, the development and implementation of a mindfulness-based exercise intervention programme aiming to decrease body image concerns and disordered eating for healthy female university students is paradoxically rare. However, an intervention to decrease dysfunctional exercise and increase mindfulness in exercising was implemented for clinical samples or patients with eating disorders and depression [52, 53, 62]. The results of those studies suggest that mindful exercising might help to decrease negative body image in patients with eating disorders. Our mindfulness-based exercise intervention programme to promote positive body image in female university students is one of the first attempts that has been made in any country. Finally, we used sound and validated instruments testing body image and related variables in this study as recommended by experts [16]. This can support the validity and reliability of our data.

Keeping in mind the enormous efforts that have been made to increase physical activity in young female, it is important to have a deeper understand of the underlying mechanisms between mindful physical activity and the development of positive body image. Thus, it is fundamental to test the efficacy of the proposed intervention programme with larger student samples. Developing and testing positive body image intervention program in men is also important [16]. Furthermore, after confirming the efficacy of the programme, it is important to test its effectiveness in real-world conditions [63].

\section{Conclusions}

The present universal education and mindfulness-based exercise intervention programme promoted a post-interventional effect on positive body image (increased body appreciation, appearance evaluation and body area satisfaction), reduction in negative body image (decreased body dissatisfaction and drive for thinness) and decreased internalisation of sociocultural stereotyped ideals in female students. The preliminary findings of this study support the efficacy of an interventional programme that incorporates cognitive behavioural therapy and mindfulness-based exercise to promote positive body image in student-aged women. Future studies should test the efficacy of the introduced programme in larger randomised samples of young women.

\section{What is already known on this subject?}

Mindfulness and positive embodiment-oriented cognitivebehavioural techniques-based educational intervention programmes targeting multiple components of positive body image have a positive effect on various aspects of body image. Most students can benefit from engagement in universal intervention programmes targeting positive body image and a healthy lifestyle. Mindful exercise might help to improve positive body image in young women. 


\section{What does this study add?}

The preliminary findings of this study support the efficacy of newly developed cognitive-behavioural therapy methods and mindful exercise-based intervention program "Body and mind" aimed to promote positive body image in student-aged women. The intervention significantly improved positive body image, reduced body dissatisfaction, drive for thinness and internalisation of stereotyped beauty ideals with medium to large effects. Future studies should test the efficacy of the introduced program in large randomised samples of young women.

Acknowledgements The authors thank all participating university students and lecturers: Simona Pajaujienè (Lithuanian Sports University), Vinga Indriūnienė (Lithuanian Sports University) and Vilma Kriaučionienè (Lithuanian University of Health Sciences). We also thank the health and fitness coaches Indre Piličiauskaite, Laura Tumynaite and Martynas Golubovskis, who implemented the mindfulness-based exercise programme. We also want to thank manager Osvaldas Duoblys for the kind support and allowing us to implement the exercise programme in the health and fitness club 'Sporthouse'.

Author contributions $\mathrm{VB}, \mathrm{RJ}$ and $\mathrm{MB}$ were involved in conceptualisation and methodology of the research. RJ supervised the scientific project. VB supervised the experiment and collected the data. MB and VB were involved in the formal statistical analyses of data. RJ, $\mathrm{VB}$ and $\mathrm{MB}$ worked on the original draft preparation and writing the manuscript. VB, RJ and MB were involved in review and editing of the final version of the manuscript.

Funding This research received no external funding.

\section{Declarations}

Conflicts of Interest The authors declare no conflict of interest.

Ethical approval All procedures performed in studies involving human participants were in accordance with the ethical standards of the institutional and/or national research committee and with the 1964 Helsinki declaration and its later amendments or comparable ethical standards.

Informed consent All participants provided informed consent prior to their participation.

Data availability statement The dataset generated and analysed during the current study is not publicly available but is available from the corresponding author upon reasonable request.

\section{References}

1. Fitzsimmons-Craft EE, Karam AM, Monterubio GE, Taylor CB, Wilfley DE (2019) Screening for eating disorders on college campuses: A review of the recent literature. Curr Psychiatry Rep 21:101-121. https://doi.org/10.1007/s11920-019-1093-1

2. Lipson SK, Jones JM, Taylor CB, Wilfley DE, Eichen DM, Fitzsimmons-Craft EE et al (2017) Understanding and promoting treatment-seeking for eating disorders and body image concerns on college campuses through online screening, prevention and intervention. Eat Behav 25:68-73. https://doi.org/10.1016/j.eatbeh.2016.03.020

3. Harrer M, Adam SH, Messner E, Baumeister H, Cuijpers P, Bruffaerts R et al (2020) Prevention of eating disorders at universities: A systematic review and meta-analysis. Int J Eat Disord 53:813833. https://doi.org/10.1002/eat.23224

4. Kass AE, Jones M, Kolko RP, Altman M, Fitzsimmons-Craft EE, Eichen DM et al (2017) Universal prevention efforts should address eating disorder pathology across the weight spectrum: Implications for screening and intervention on college campuses. Eat Behav 25:74-80. https://doi.org/10.1016/j.eatbeh.2016.03.019

5. McVey GL, Kirsh G, Maker D, Walker KS, Mullane J, Laliberte $M$ et al (2010) Promoting positive body image among university students: A collaborative pilot study. Body Image 7:200-204. https://doi.org/10.1016/j.bodyim.2010.02.005

6. Alleva JM, Sheeran P, Webb TL, Martijn C, Miles E (2015) A meta-analytic review of stand-alone interventions to improve body image. PLoS ONE 10:e0139177. https://doi.org/10.1371/journal. pone. 0139177

7. Dakanalis A, Clerici M, Stice E (2019) Prevention of eating disorders: current evidence-base for dissonance-based programmes and future directions. Eat Weight Disord 24:597-603. https://doi. org/10.1007/s40519-019-00719-3

8. Ciao AC, Loth K, Neumark-Sztainer D (2014) Preventing eating disorder pathology: Common and unique features of successful eating disorders prevention programs. Curr Psychiatry Rep 16:453-476. https://doi.org/10.1007/s11920-014-0453-0

9. Levine MP, Smolak L (2007) Prevention of negative body image, disordered eating, and eating disorders: an update. In: Wonderlich S, Mitchell JE, de Zwaan M, Steiger H, F van Furth E (ed) Annual Review of Eating Disorders: Part 1. CRC Press, London pp 1-13

10. Yager Z, O'Dea JA (2008) Prevention programs for body image and eating disorders on University campuses: a review of large, controlled interventions. Health Promot Int 23:173-189. https:// doi.org/10.1093/heapro/dan004

11. Piran N (2017) Journeys of embodiment at the intersection of body and culture: The developmental theory of embodiment. Academic Press, San Diego

12. Tylka TL, Wood-Barcalow NL (2015) What is and what is not positive body image? Conceptual foundations and construct definition. Body Image 14:118-129. https://doi.org/10.1016/j.bodyim. 2015.04.001

13. Tylka TL, Wood-Barcalow N (2015) The Body Appreciation Scale-2: item refinement and psychometric evaluation. Body Image 12:53-67. https://doi.org/10.1016/j.bodyim.2014.09.006

14. Alleva JM, Tylka TL (2021) Body functionality: A review of the literature. Body Image 36:149-171. https://doi.org/10.1016/j. bodyim.2020.11.006

15. Atkinson MJ, Stock NM, Alleva JM, Jankowski GS, Piran N, Riley S et al (2020) Looking to the future: Priorities for translating research to impact in the field of appearance and body image. Body Image 32:53-61. https://doi.org/10.1016/j.bodyim.2019.10. 006

16. Guest E, Costa B, Williamson H, Meyrick J, Halliwell E, Harcourt D (2019) The effectiveness of interventions aiming to promote positive body image in adults: A systematic review. Body Image 30:10-25. https://doi.org/10.1016/j.bodyim.2019.04.002

17. Levine MP, Smolak L (2015) The role of protective factors in the prevention of negative body image and disordered eating. Eat Disord 24:39-46. https://doi.org/10.1080/10640266.2015.11138 26

18. Sundgot-Borgen C, Bratland-Sanda S, Engen KM, Pettersen G, Friborg O, Torstveit MK et al (2018) The Norwegian healthy body image programme: study protocol for a randomized controlled school-based intervention to promote positive body 
image and prevent disordered eating among Norwegian high school students. BMC Psychol 6:1-9. https://doi.org/10.1186/ s40359-018-0221-8

19. Sundgot-Borgen C, Friborg O, Kolle E, Engen KM, Sundgot-Borgen J, Rosenvinge JH et al (2019) The healthy body image (HBI) intervention: Effects of a school-based cluster-randomized controlled trial with 12-months follow-up. Body Image 29:122-131. https://doi.org/10.1016/j.bodyim.2019.03.007

20. Annesi J (2005) Relations of body esteem factors with exercise session attendance in women initiating a physical activity program. Percept Mot Skills 100:995-1003. https://doi.org/10.2466/ pms.100.3c.995-1003

21. Pinto BM, Clark MM, Maruyama NC, Feder SI (2003) Psychological and fitness changes associated with exercise participation among women with breast cancer. Psychooncology 12:118-126. https://doi.org/10.1002/pon.618

22. Sabiston CM, Pila E, Vani M, Thogersen-Ntoumani C (2019) Body image, physical activity, and sport: A scoping review. Psychol Sport Exerc 42:48-57. https://doi.org/10.1016/j.psychsport. 2018.12.010

23. Tylka TL, Homan KJ (2015) Exercise motives and positive body image in physically active college women and men: Exploring an expanded acceptance model of intuitive eating. Body Image 15:90-97. https://doi.org/10.1016/j.bodyim.2015.07.003

24. Hurst M, Dittmar H, Banerjee R, Bond R (2017) "I just feel so guilty": The role of introjected regulation in linking appearance goals for exercise with women's body image. Body Image 20:120 129. https://doi.org/10.1016/j.bodyim.2016.12.002

25. Calogero RM, Tylka TL, McGilley BH, Pedrotty-Stump KN (2019) Attunement with exercise (AWE). In: Tylka TL, Piran N (eds) Handbook of positive body image and embodiment: Constructs, protective factors, and interventions. Oxford University Press, New York, pp 80-90

26. Augustus-Horvath CL, Tylka TL (2011) The acceptance model of intuitive eating: A comparison of women in emerging adulthood, early adulthood, and middle adulthood. J Couns Psychol 58:110-125. https://doi.org/10.1037/a0022129

27. Reel JJ, Galli N, Miyairi M, Voelker D, Greenleaf C (2016) Development and validation of the intuitive exercise scale. Eat Behav 22:129-132. https://doi.org/10.1016/j.eatbeh.2016.06.013

28. Piran N (2019) The experience of embodiment construct: Reflecting the quality of embodied lives. In: Tylka TL, Piran N (eds) Handbook of positive body image and embodiment: Constructs, protective factors, and interventions. Oxford University Press, New York, pp 11-21

29. Tiggemann M, Zaccardo M (2015) "Exercise to be fit, not skinny": The effect of fitspiration imagery on women's body image. Body Image 15:61-67. https://doi.org/10.1016/j.bodyim.2015.06.003

30. Homan KJ, Tylka TL (2014) Appearance-based exercise motivation moderates the relationship between exercise frequency and positive body image. Body Image 11:101-108. https://doi.org/10. 1016/j.bodyim.2014.01.003

31. Cox AE, Ullrich-French S, Tylka TL, McMahon AK (2019) The roles of self-compassion, body surveillance, and body appreciation in predicting intrinsic motivation for physical activity: Crosssectional associations, and prospective changes within a yoga context. Body Image 29:110-117. https://doi.org/10.1016/j.bodyim. 2019.03.002

32. Menzel JE, Thompson JK, Levine MP (2019) Development and validation of the physical activity body experiences questionnaire. Bull Menninger Clin 83:53-83. https://doi.org/10.1521/bumc. 2019.83.1.53

33. Neumark-Sztainer D, MacLehose RF, Watts AW, Pacanowski CR, Eisenberg ME (2018) Yoga and body image: Findings from a large population-based study of young adults. Body Image 24:69-75. https://doi.org/10.1016/j.bodyim.2017.12.003
34. Alleva JM, Tylka TL, van Oorsouw K, Montanaro E, Perey I, Bolle C et al (2020) The effects of yoga on functionality appreciation and additional facets of positive body image. Body Image 34:184-195. https://doi.org/10.1016/j.bodyim.2020.06.003

35. Halliwell E, Dawson K, Burkey S (2019) A randomized experimental evaluation of a yoga-based body image intervention. Body Image 28:119-127. https://doi.org/10.1016/j.bodyim.2018.12.005

36. Baceviciene M, Jankauskiene R, Balciuniene V (2020) The role of body image, disordered eating and lifestyle on the quality of life in lithuanian university students. Int J Environ Res Public Health 17:1593. https://doi.org/10.3390/ijerph17051593

37. Dhand N, Khatkar M (2014) Statulator: An online statistical calculator. Sample size calculator for comparing paired differences. http://statulator.com/SampleSize/ss2PM.html. Accessed 1 Feb 2021

38. Schaefer LM, Burke NL, Thompson JK, Dedrick RF, Heinberg LJ, Calogero RM et al (2015) Development and validation of the sociocultural attitudes towards appearance questionnaire-4 (SATAQ-4). Psychol Assess 27:54-67. https://doi.org/10.1037/ a0037917

39. Baceviciene M, Jankauskiene R, Balciuniene V (2020) Validation of the lithuanian version of the sociocultural attitudes towards appearance questionnaire-4 (SATAQ-4) in a student sample. Int J Environ Res Public Health 17:932. https://doi.org/10.3390/ijerp h17030932

40. Brown TA, Cash TF, Mikulka PJ (1990) Attitudinal body-image assessment: factor analysis of the body-self relations questionnaire. J Pers Assess 55:135-144. https://doi.org/10.1080/00223 891.1990.9674053

41. Miskinyte A, Bagdonas A (2010) Body image relationship with demographic variables in young adults. Psichologija 42:85-101

42. Garner DM (2004) Eating Disorder Inventory-3: Professional Manual. Psychological Assessment Resources, Lutz

43. Baceviciene M, Jankauskiene R (2020) Associations between body appreciation and disordered eating in a large sample of adolescents. Nutrients 12:752. https://doi.org/10.3390/nu12030752

44. World Health Organization (1997) Obesity: preventing and managing the global epidemic: report of a WHO consultation on obesity, No. WHO/NUT/NCD/98.1. WHO, Geneva, Switzerland

45. Godin G, Shephard RJ (1985) A simple method to assess exercise behavior in the community. Can J Appl Sport Sci 10:141-146

46. Fairburn CG, Beglin SJ (1994) Assessment of eating disorders: Interview or self-report questionnaire? Int J Eat Disord 16:363-370

47. Baceviciene M, Balciuniene V, Jankauskiene R (2020) Validation of the lithuanian version of the eating disorder examination questionnaire 6.0 in a student sample. Brain Behav 10:e001555. https://doi.org/10.1002/brb3.1555

48. Reeder LG, Chapman JM, Coulson AH (1968) Socioenvironmental stress, tranquilizers and cardiovascular disease. Proce Excerpta Medica Intern Congress Ser 182:226-238

49. Markeviciute A (2003) Psychosocial stress correlations with depressiveness among adolescents. Biologine psichiatrija ir psichofarmakologija 5:11-14

50. Leppink J, O'sullivan P, Winston K, (2017) Are differences between groups different at different occasions? Perspect Med Educ 6:413-417. https://doi.org/10.1007/s40037-017-0380-y

51. Greenleaf C, Hauff C (2019) Environments that Cultivate Positive Embodiment Through Mindful Movement. In: Tylka TL, Piran N (eds) Handbook of Positive Body Image and Embodiment: Constructs, Protective Factors, and Interventions. Oxford University Press, New York, pp 118-128

52. Calogero RM, Pedrotty KN (2004) The practice and process of healthy exercise: an investigation of the treatment of exercise abuse in women with eating disorders. Eat Disord 12:273-291. https://doi.org/10.1080/10640260490521352 
53. Tsang HW, Chan EP, Cheung WM (2008) Effects of mindful and non-mindful exercises on people with depression: a systematic review. Br J Clin Psychol 47:303-322. https://doi.org/10.1348/ $014466508 X 279260$

54. Tiggemann M, Coutts E, Clark L (2014) Belly dance as an embodying activity?: A test of the embodiment model of positive body image. Sex Roles 71:197-207. https://doi.org/10.1007/ s11199-014-0408-2

55. Jankauskiene R, Baceviciene M, Trinkuniene L (2020) Examining body appreciation and disordered eating in adolescents of different sports practice: cross-sectional study. Int J Environ Res Public Health 17:4044. https://doi.org/10.3390/ijerph17114044

56. Soulliard ZA, Kauffman AA, Fitterman-Harris HF, Perry JE, Ross MJ (2019) Examining positive body image, sport confidence, flow state, and subjective performance among student athletes and non-athletes. Body Image 28:93-100. https://doi.org/10.1016/j. bodyim.2018.12.009

57. Rodgers RF, Lombardo C, Cerolini S, Franko DL, Omori M, Fuller-Tyszkiewicz M et al (2020) The impact of the COVID-19 pandemic on eating disorder risk and symptoms. Int J Eat Disord 53:1166-1170. https://doi.org/10.1002/eat.23318

58. Swami V, Horne G, Furnham A (2021) COVID-19-related stress and anxiety are associated with negative body image in adults from the United Kingdom. Pers Individ Differ 170:110426. https:// doi.org/10.1016/j.paid.2020.110426

59. Tylka TL, Annunziato RA, Burgard D, Daníelsdóttir S, Shuman E, Davis C et al (2014) The weight-inclusive versus weight-normative approach to health: Evaluating the evidence for prioritizing well-being over weight loss. J Obes. https://doi. org/10.1155/2014/983495

60. Sánchez-Carracedo D, Neumark-Sztainer D, López-Guimerà G (2012) Integrated prevention of obesity and eating disorders: barriers, developments and opportunities. Public Health Nutr 15:2295-2309. https://doi.org/10.1017/S1368980012000705

61. Leme ACB, Thompson D, Dunker KLL, Nicklas T, Philippi ST, Lopez T et al (2018) Obesity and eating disorders in integrative prevention programmes for adolescents: protocol for a systematic review and meta-analysis. BMJ Open 8:e020381. https://doi.org/ 10.1136/bmjopen-2017-020381

62. Calogero RM, Pedrotty KN (2010) Incorporating exercise into the treatment and recovery of eating disorders: Cultivating a mindful approach. In: Maine M, Bunnell D, McGilley BH (eds) Treatment of eating disorders: Bridging the research-practice gap. Elsevier, New York, pp 425-441

63. Singal AG, Higgins PD, Waljee AK (2014) A primer on effectiveness and efficacy trials. Clin Transl Gastroenterol 5:e45. https:// doi.org/10.1038/ctg.2013.13

Publisher's Note Springer Nature remains neutral with regard to jurisdictional claims in published maps and institutional affiliations. 